\title{
The European Training Network ETUDE (Encompassing Training in Functional Disorders across Europe) Is Recruiting 15 Early-Stage Researchers
}

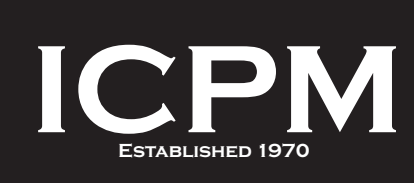
J.G.M. Rosmalen ${ }^{\mathrm{a}, \mathrm{i}}$
C. Burton ${ }^{b}$
A. Carson ${ }^{c}$
Fiammetta Coscid
L. Frostholm ${ }^{\mathrm{e}}$
N. Lehnen ${ }^{f}$ T. Olde Hartman ${ }^{g}$
C. Rask ${ }^{\mathrm{e}}$
J. Rymaszewska ${ }^{\text {h }}$
J. Stone ${ }^{c}$
L. Tak ${ }^{i}$
M. Witthöft ${ }^{\mathrm{j}}$
B. Löwe ${ }^{k}$

a Departments of Psychiatry and Internal Medicine, University Medical Center Groningen, Groningen,

The Netherlands; ${ }^{\mathrm{b}}$ Academic Unit of Primary Medical Care, University of Sheffield, Sheffield, UK; ' Neurology,

Centre for Clinical Brain Sciences, University of Edinburgh, Edinburgh, UK; ${ }^{d}$ Department of Health Sciences,

University of Florence, Florence, Italy; ${ }^{\mathrm{e}}$ Research Clinic Functional Disorders, Aarhus University Hospital, Aarhus,

Denmark; ${ }^{\mathrm{f} D e p a r t m e n t}$ of Psychosomatic Medicine and Psychotherapy, Technische Universität München,

Munich, Germany; ${ }^{9}$ Radboud Institute for Health Sciences, Radboud University Medical Center Nijmegen, Nijmegen,

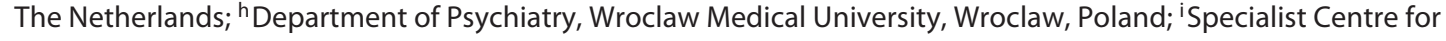

Functional Symptoms and Somatic Symptom Disorders, Dimence Mental Health Care, Deventer, The Netherlands;

jDepartment of Clinical Psychology, Psychotherapy, and Experimental Psychopathology,

Johannes Gutenberg-University Mainz, Mainz, Germany; ${ }^{\mathrm{k}}$ Department of Psychosomatic Medicine and

Psychotherapy, University Medical Center Hamburg-Eppendorf, Hamburg, Germany

Functional disorders are traditionally understood as clusters of persistent somatic symptoms that have typical clinical characteristics but currently cannot be associated to reproducibly observable pathophysiological mechanisms. Knowledge on functional disorders is limited and fragmented, leading to insufficient health care for patients with functional disorders, and a society in which patients with functional disorders experience stigma from both the society as well as from health care professionals.

In response to these challenges, the EURONET-SOMA network developed a sustainable and structured training programme aimed at educating a new generation of interdisciplinary creative early stage researchers (ESR) that are able to cross disciplines and to translate theory and experimental models to products and services that improve care for patients. This will be done in the form of the European Training Network "Encompassing Training in fUnctional Disorders across Europe (ETUDE)“" which has received funding from the European Union's Horizon 2020 research and innovation programme under the Marie Skłodowska-Curie grant agreement No. 956673.

Research within ETUDE is organized into four research work packages. In the first, four PhD students will work on transdiagnostic mechanisms underlying the development of functional disorders. The second research work package is focused on diagnosis of functional disorders, and will involve three $\mathrm{PhD}$ students who will study predictive validity of diagnostic criteria. The third research work package involves five $\mathrm{PhD}$ students focusing on treatment of functional disorders. In the fourth research work package, three $\mathrm{PhD}$ students will study stigma, and develop and evaluate methods and products to reduce it. karger@karger.com

(c) 2021 S. Karger AG, Basel

www.karger.com/pps

Karger!
Fiammetta Cosci

Psychology and Psychiatry Unit, Department of Health Sciences

University of Florence, Via di San Salvi, 12

IT-50135 Florence (Italy)

fiammetta.cosci@unifi.it 
Central training within ETUDE is organized into network-wide training schools, each involving an advanced scientific course on a specific topic related to functional disorders, a transferable skill course focusing on general academic skills, and an outreach activity. The topics of the training schools follow the work package structure. The training schools will also be open to $\mathrm{PhD}$ students that perform their research outside the ETUDE programme.
ETUDE is currently recruiting $15 \mathrm{PhD}$ students for this European research programme and training network. For a more extensive description of ETUDE we refer to a recent publication (https://www.sciencedirect.com/science/ article/pii/S0022399920309077). Details on the specific projects, recruitment procedures, eligibility criteria and on how to apply can be found at https://www.euronetsoma.eu/itn/. For further information on the research or training programme you can contact etude@umcg.nl. 